\title{
Highly dispersed PVP-supported Ir-Ni bimetallic nanoparticles as high performance catalyst for degradation of metanil yellow
}

\author{
ANJALI GOEL and MANU CHAUDHARY* \\ Department of Chemistry, KGC, Gurukul Kangri University, Haridwar 249407, India \\ *Author for correspondence (manuchaudhary349@gmail.com)
}

MS received 14 July 2017; accepted 9 October 2017; published online 23 May 2018

\begin{abstract}
Bimetallic nanoparticles, a new class of materials for catalysis, were intensively investigated. Highly dispersed Ir-Ni bimetallic nanoparticles with varying mole fractions were synthesized by modified polyol reduction method from the solution of iridium trichloride and nickel chloride in ethylene glycol, which acts as both solvent and reducing agent. The particles were characterized for their size, morphology and composition using various techniques like UV-Vis, Fourier transform infrared (FT-IR), X-ray diffraction (XRD), transmission electron microscopy (TEM), field emission scanning electron microscopy (FE-SEM) and X-ray photo electron spectroscopic (XPS) techniques. The synthesized nanoparticles were in pseudo-spherical morphology and utilized as catalyst for the degradation of dyes. The feasibility of degradation of an azo dye i.e., metanil yellow (MY) in aqueous alkaline medium by hexacynoferrate (III) ions using Ir-Ni bimetals as catalyst, prepared in different molar ratios, was investigated. Results show that Ir-Ni (2:1) bimetals have good catalytic activity and degradation as compared to $\mathrm{Ir}-\mathrm{Ni}$ (1:2) due to their small size and high stability for the oxidation of MY.
\end{abstract}

Keywords. Ethylene glycol; iridium trichloride; nickel chloride; polyvinyl pyrrolidone (PVP); metanil yellow (MY); degradation.

\section{Introduction}

Fast increasing population, fast pace of industrialization, modernization of civilizations, agricultural as well as domestic wastes and several environmental changes are declining the quality of water [1]. Textile dyes and other commercial colourants have become a major concern to environmental pollution. Many dyes are made as highly water-soluble to meet the colour requirement of deep dyeing. As a consequence, traditionally, wastewater becomes contaminated [2]. Waste water from textile, paper, food and some other industries contain residual dyes, which are not readily biodegradable. Azo dyes possess one or more azo groups and are widely used in many industries because of their chemical stability and its comparatively easier synthetic processes [3]. The effluent streams from textile plants are highly coloured in most cases. The depolarization of this kind of wastewater becomes very important not only for aesthetic reason, but also because many dyes and their breakdown products are toxic to aquatic life and mutagenic to humans [4]. Metanil yellow (MY), an azo dye was proved to be heptatoxic in albino rats $[5,6]$. Thus, it is very essential to degrade dyes and make the environment free from toxicity. A wide range of methods were developed for the removal of synthetic dyes from waters and wastewaters to decrease their impact on the environment. The technologies involve adsorption on inorganic or organic matrices, decolourization by photocatalysis, and/or by oxidation processes, microbiological or enzymatic decomposition, etc. The efficacy of various methods of dye removal, such as chemical precipitation, chemical oxidation, adsorption along with their effects on subsequent biological treatment was compared in an earlier paper, which suggest that the chemical oxidation is the effective method, but the efficiency strongly influenced by the type of oxidant used [7].

Some more methods are coagulation, flocculation, reverse osmosis and adsorption on activated carbon [8]. In the past few years, it was observed that the nanoparticles were also used for the oxidation of several dyes [9].

The interest in the study and procedure of nanoscale bimetallic nanoparticles increased nowadays. Various bimetallic nanoparticles were synthesized in the recent past because of the modification of property not only due to size effects, but also as a result of incorporation of second catalytic metal e.g., Pd, Ni and Pt modifies the catalytic, electronic and optical properties and enhance degradation rates as compared to nanoiron alone [10-12]. When a metal is associated with another metal in bimetallic or alloy form, the properties of the resulting material can be enhanced with respect to those of pure metals. Metal nanoparticle catalysts are highly demanded for various indisiciplinary chemical processes. A huge number of metal nanoparticles were developed by reduction of metal salt precursors in the presence of surfactants, which prevents the nanoparticles from aggregation in the solution-phase synthetic methods [13-15].

Among the various metal and metal oxide nanoparticles studied so far, the transition metal oxides are an important 
group of materials as they form a wide variety of structures, display many interesting properties, and have numerous applications. They also show fascinating colour change in the $\mathrm{UV}-\mathrm{Vis}$ region due to their close lying conduction and valence bands in which electrons can move freely [16]. Among these, iridium is particularly an interesting transition metal catalyst. There are reports of unusually high catalytic activity from molecular iridium cluster [16,17]. The use of nickel-based catalyst is common in chemical industries due to their lowcost and active catalyst as those of Pt, Rh, Pd, Ru and Ir. It can be seen that transition metal nanoparticles are very attractive to use as catalyst due to their high surface to volume ratio and their high surface energy, which make their surface atom active $[18,19]$. Many techniques and methods were adopted to prepare bimetallic nanoparticles of various kinds, including alcohol reduction, seed growth method, polyol process, solvent extraction-reduction, sono chemical method, photocatalytic reduction, environmentally begin synthesis process and metal evaporation condensation [20].

Bimetallic ultrafine particle often agglomerate to form either lumps or secondary particle to minimize the total surface or the interfacial energy of the system, therefore, it is very important to stabilize the particle against adverse agglomeration at both the synthesis and the usage stage. In this paper, we have synthesized bimetallic Ir-Ni nanoparticles with $(2: 1)$ and (1:2) molar ratio by modified polyol method, using polyvinyl pyrrolidone (PVP) as capping agent. These particles were characterized by UV-Vis, Fourier transform infrared (FT-IR), transmission electron microscopy (TEM), field emission scanning electron microscopy (FE-SEM), $\mathrm{X}$-ray diffraction (XRD) and X-ray photo electron spectroscopic (XPS) methods of analysis.

\section{Experimental}

\subsection{Materials}

All reagents were obtained from typical commercial sources and used without further treatment. Iridium trichloride, nickel chloride (used as precursor salt) and ethylene glycol (used as solvent and reducing agent) were purchased from SRL. Sodium hydroxide pellets were obtained from Merck. PVP (SRL, MW 10,000) was used as capping agent. All solutions were prepared in double-distilled water.

\subsection{Equipments}

UV-Vis spectra during the synthesis were recorded using systronic-117 spectrophotometer in the range of 220-550 nm. The FT-IR spectra were measured with a Perkin Elmer using the $\mathrm{KBr}$ pellet technique. The XRD measurements were conducted by the dry powders using Bruker Axs D- 8 advance diffractometer with $\mathrm{CuK} \alpha(\lambda=0.154 \mathrm{~nm})$ radiation over a range of $2 \theta$ angles from 20 to $90^{\circ}$. The average thickness of the sample was determined using the Debye Scherrer equation. Transmission electron micrographs were obtained using TEM CM 200, operated at an accelerating voltage of 20-200 $\mathrm{kV}$. Randomly orientated particle on the TEM substrate were obtained by using diluted solution of nanoparticles. For surface morphology, Zeiss EVO-18 SEM was used. The surface composition and oxidation state of nanoparticles were analysed by XPS using a PHI 5000 Versa Prob II, FEI Inc. using Al monochromatic X-ray at a power of $24.6 \mathrm{~W}$ and the spectra were obtained at electron take off angle $45^{\circ}$ by passing the energy of $187.85 \mathrm{eV}$.

\subsection{Synthesis of bimetallic nanoparticles}

In the present work, synthesis of $\mathrm{Ir}-\mathrm{Ni}$ bimetllic nanoparticles in different molar ratios $(4: 1,1: 4,3: 1,1: 3,2: 1,1: 2$, $1: 1)$ using precursor salts of iridium chloride and nickel chloride by modified polyol reduction method was carried out [21]. The synthesis was monitored spectrophotometrically. A constant peak in the spectrum is the indication of formation of stable bimetallic nanoparticles. The synthesis of Ir:Ni bimetallic nanoparticles in $4: 1,1: 4,3: 1$ and $1: 3$ ratios do not show any constant peaks, but $1: 2$ and $2: 1$ show constant peaks at 324 and $342 \mathrm{~nm}$, respectively (table 1). Polymer-stabilized bimetallic nanoparticles in alcohol were prepared by simultaneous reduction of the two corresponding metal salts. Precursor salts of iridium trichloride and nickel

Table 1. Synthesis conditions for the preparation of (Ir:Ni) bimetallic nanoparticles.

\begin{tabular}{|c|c|c|c|c|c|c|}
\hline $\mathrm{IrCl}_{3}(\mathrm{M})$ & $\mathrm{NiCl}_{2} \cdot 5 \mathrm{H}_{2} \mathrm{O}(\mathrm{M})$ & Ir:Ni & $\operatorname{PVP}(\mathrm{M})$ & Ethylene glycol & $\mathrm{NaOH}$ & $\lambda_{\max }$ \\
\hline $1.182 \times 10^{-3}$ & $4.9 \times 10^{-3}$ & $1: 4$ & $7 \times 10^{-6}$ & $25 \mathrm{ml}$ & $1 \mathrm{ml}$ & No stable peak \\
\hline $3.9 \times 10^{-3}$ & $0.98 \times 10^{-3}$ & $4: 1$ & $7 \times 10^{-6}$ & $25 \mathrm{ml}$ & $1 \mathrm{ml}$ & No stable peak \\
\hline $1.182 \times 10^{-3}$ & $3.9 \times 10^{-3}$ & $1: 3$ & $7 \times 10^{-6}$ & $25 \mathrm{ml}$ & $1 \mathrm{ml}$ & No stable peak \\
\hline $2.9 \times 10^{-3}$ & $0.98 \times 10^{-3}$ & $3: 1$ & $7 \times 10^{-6}$ & $25 \mathrm{ml}$ & $1 \mathrm{ml}$ & No stable peak \\
\hline $1.182 \times 10^{-3}$ & $2.4 \times 10^{-3}$ & $1: 2$ & $7 \times 10^{-6}$ & $25 \mathrm{ml}$ & $1 \mathrm{ml}$ & $324 \mathrm{~nm}$ \\
\hline $0.98 \times 10^{-3}$ & $1.9 \times 10^{-3}$ & $2: 1$ & $7 \times 10^{-6}$ & $25 \mathrm{ml}$ & $1 \mathrm{ml}$ & $342 \mathrm{~nm}$ \\
\hline $1.182 \times 10^{-3}$ & $0.98 \times 10^{-3}$ & $1: 1$ & $7 \times 10^{-6}$ & $25 \mathrm{ml}$ & $1 \mathrm{ml}$ & $279 \mathrm{~nm}$ \\
\hline
\end{tabular}

Bold indicates the working experimental conditions. 


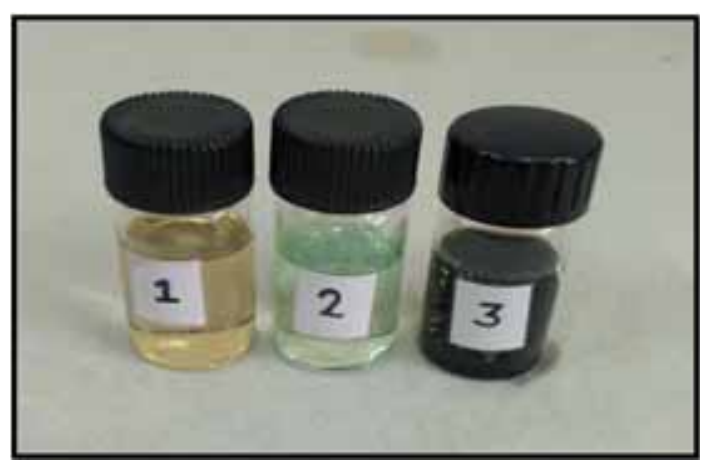

Figure 1. $(1,2,3)$ The colour of iridium precursor, nickel precursor and their bimetallic nanoparticles, respectively.

chloride in different molar ratios were dissolved in $25 \mathrm{ml}$ ethylene glycol. A certain amount of PVP was added to this solution and the system was maintained under magnetic stirrer until total dissociation of PVP occurs. Required amount of $\mathrm{NaOH}$ is added by maintaining $\mathrm{pH} 11$. After being stirred at room temperature, the whole content was transferred into three-necked flask and reflux for $2 \mathrm{~h}$ in oil bath at 140 $180^{\circ} \mathrm{C}$ temperature. After refluxing, the colour of the solution becomes blackish brown from pale yellow (figure 1). The synthesized colloidal dispersion is stable and it neither precipitates nor flocculates over a period of several months.

\section{Results and discussion}

The formation of Ir-Ni bimetallic nanoparticles was noticeable by the dramatic colour change of the system from pale yellow to brown. In general, reaction proceeds as an oxidation of the ethylene glycol reducing the metallic precursor to its zero-valent state. The overall reaction based on the experimental results and previously reported work, the following reaction mechanism can be proposed for the synthesis of bimetallic Ir-Ni nanoparticles [22]:

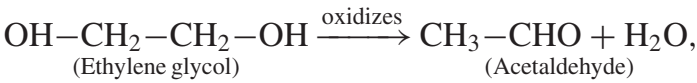

$$
\begin{aligned}
& 6\left(\mathrm{CH}_{3}-\mathrm{CHO}\right)+2 \mathrm{IrCl}_{3}
\end{aligned}
$$

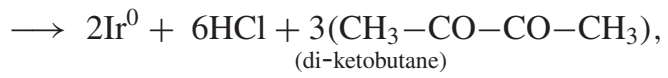

$$
\begin{aligned}
& 2\left(\mathrm{CH}_{3}-\mathrm{CHO}\right)+\mathrm{NiCl}_{2} \cdot 6 \mathrm{H}_{2} \mathrm{O} \\
& \longrightarrow \mathrm{Ni}^{0}+2 \mathrm{HCl}+\underset{\text { (di-ketobutane) }}{6 \mathrm{CH}_{2} \mathrm{O}}-\mathrm{CO}-\mathrm{CO}-\mathrm{CH}_{3} .
\end{aligned}
$$

The above reactions explain the reduction of $\operatorname{Ir}^{+3}$ to $\operatorname{Ir}^{0}$ and $\mathrm{Ni}^{+2}$ to $\mathrm{Ni}^{0}$.

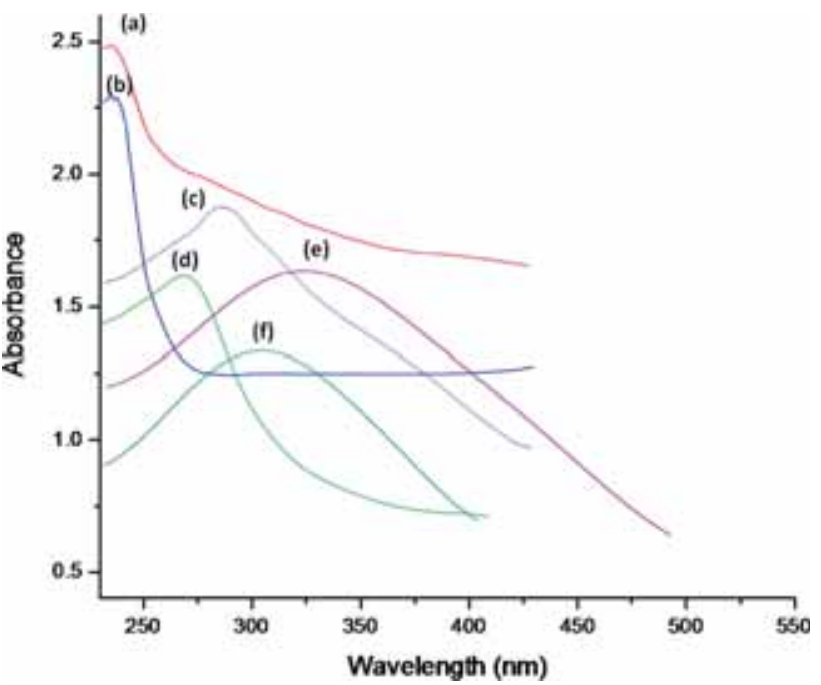

Figure 2. UV-Vis spectra of (a) iridium precursor, (b) nickel precursor, (c) iridium(0) monometal, (d) nickel(0) monometal, (e) Ir :Ni (2:1) bimetals and (f) Ir:Ni (1:2) bimetals.

\subsection{UV-Vis spectrophotometry}

UV-Vis spectrophotometer was used to carry out the optical measurements. The sample was placed in quartz cuvette using ethylene glycol as a reference solution. The absorption spectra of precursor salt of $\mathrm{Ir}, \mathrm{Ni}$ and monometallic and bimetallic nanoparticles of $\mathrm{Ir}$ and $\mathrm{Ni}$ in various molar ratios of $\mathrm{Ir}-\mathrm{Ni}$ are shown in figure 2. The figure shows that peaks of $\operatorname{Ir}(\mathrm{III})$ and $\mathrm{Ni}(\mathrm{II})$ are at 234 and $224.4 \mathrm{~nm}$, which on reduction by ethylene glycol shifted to 308.8 and $283.2 \mathrm{~nm}$ for $\operatorname{Ir}(0)$ monometallic and $\mathrm{Ni}(0)$ monometallic nanoparticles, respectively $[23,24]$. So two absorption bands would be aspected for a physical mixture of Ir and Ni monometallic nanoparticles and the formation of Ir-Ni alloy could be deduced from the fact that the optical absorption spectra show only one broad peak. In the spectra (e) and (f), the formation of new broad peak at 342 and $324 \mathrm{~nm}$ for Ir-Ni (2:1) and Ir-Ni (1:2), respectively, shows the formation of bimetallic nanoparticles. The spectrum of bimetallic nanoparticles is not a simple sum of those of two nanoparticles indicating that these bimetallic nanoparticles have an alloy structure. It was observed that the maximum absorption peak have shown shift from monometallic Ir and $\mathrm{Ni}$ absorption peaks depending upon their molar ratios i.e., for Ir:Ni (2:1), it shows a more shift than Ir:Ni (1:2) nanoparticles.

\section{$3.2 X R D$}

The XRD pattern of bimetallic nanoparticles was obtained

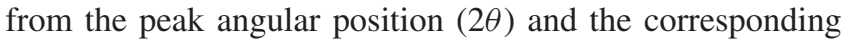
miller indices $(h k l)$ using Bragg's law.

$$
d_{\mathrm{hkl}}=n \lambda / 2 \sin \theta
$$




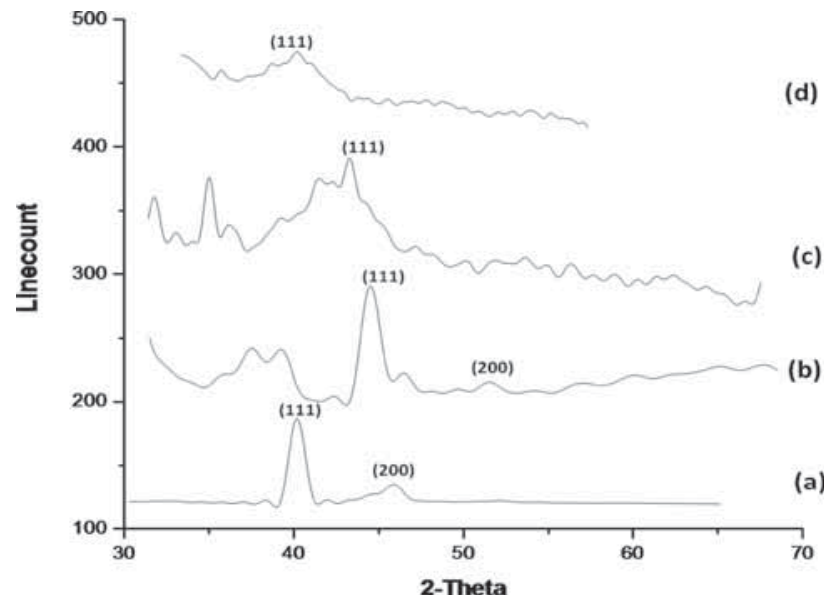

Figure 3. XRD pattern of (a) iridium monometals, (b) $\mathrm{Ni}$ monometals, (c) Ir:Ni-1:2 bimetals and (d) Ir:Ni-2:1 bimetals.

where $\lambda$ is XRD wavelength, $\theta$ the peak for $(h k l)$ each.

The average crystalline size was calculated using Debye Scherrer equation and the obtained results are listed as

$$
D=\frac{0.9 \lambda}{\cos \theta \times B},
$$

where $D$ is the average crystallite site, $B$ the full-width at half maxima, $\theta$ the Bragg angle and $\lambda$ is radiation wavelength.

The XRD spectrum of monometals of nickel, iridium and Ir-Ni bimetals in different molar ratios are shown in figure 3 . Two characteristic peaks of nickel nanoparticles $(2 \theta=45.10$, $\left.51.40^{\circ}\right)$ marked by indices $(111,200)$ were observed in figure $2 \mathrm{~b}$. This reveals that the resultant nanoparticles are of pure $\mathrm{Ni}(0)$ and with FCC structure [25]. Figure 3a shows two diffraction peaks located at $2 \theta=40.19,47.27^{\circ}$, which correspond to $\operatorname{Ir}(0)$ with crystallographic planes $(111)$ and (200), respectively (card no. JCPDS-ICDD 6-598). The lattice parameter calculated for Ir and $\mathrm{Ni}$ are 3.88 and $3.47 \AA$, respectively. The most representative reflection of Ir nanoparticles were indexed as a FCC lattice. The XRD pattern points to the formation of Ir-Ni solid solution alloy nanoparticles, possibly with a FCC structure with a lattice parameter of 3.90 and $3.54 \AA$ for Ir:Ni (2:1) and Ir:Ni (1:2), respectively. The characteristic peak for bimetals Ir: $\mathrm{Ni}(2: 1)$ were observed at $2 \theta=40^{\circ}$, which is towards Ir monometals due to the high concentration of Ir, while Ir: Ni $(1: 2)$ peak at $\left(2 \theta=44.18^{\circ}\right)$ towards $\mathrm{Ni}$ monometals due to high concentration of Ni. No separate peaks for Ir and Ni were observed in bimetallic nanoparticles. The approximate diameter calculated by Scherrer equation for Ir:Ni (2:1) and Ir: Ni (1:2) bimetals are 8.46 \pm 0.1 and 10.79 $\pm 0.1 \mathrm{~nm}$, respectively.

\section{$3.3 \quad F T-I R$}

FT-IR spectroscopy can provide vital information regarding surface interaction. Figure 4 shows that the FT-IR spectra

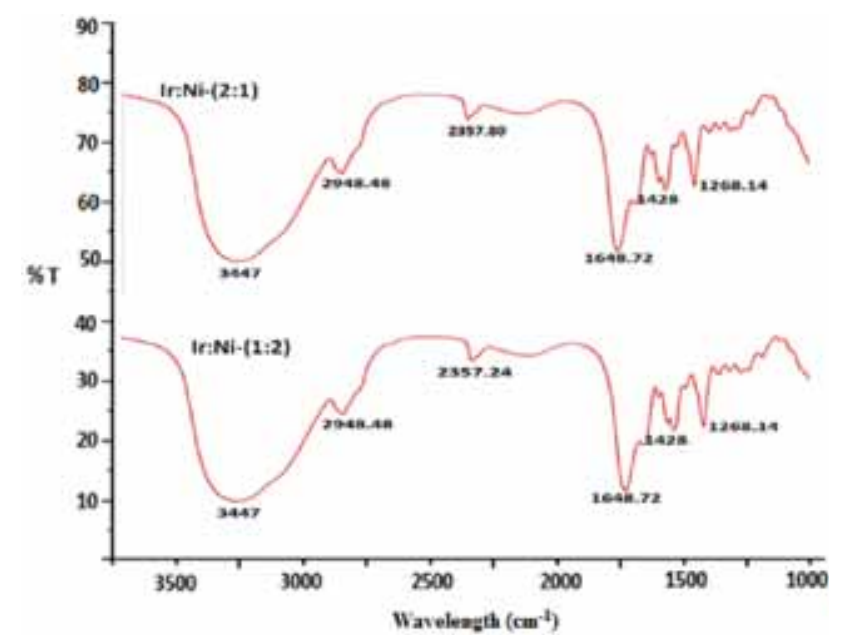

Figure 4. FT-IR spectra of Ir:Ni (2:1) and Ir:Ni (2:1) bimetallic nanoparticles.

of Ir:Ni (2:1) and Ir:Ni (1:2) bimetallic nanoparticles are almost similar types, which may be due to the involvement of same type of interaction amongst iridium, nickel and PVP molecule irrespective of $\mathrm{Ir} / \mathrm{Ni}$ ratio. The appearance of band at $2357.24 \mathrm{~cm}^{-1}$ due to the coordination of precursor salts of iridium, nickel, and PVP indicating the formation of Ir:Ni bimetallic nanoparticles. It is obvious that the surface of bimetallic nanoparticles is observed with PVP and the chemical interaction with PVP and bimetals took place based on the shifting of carbonyl bond absorption peak from 1644 for pure PVP to 1648 for bimetallic nanoparticles supported by PVP [26]. In all the spectra, the broad absorption band also appeared at $1428 \mathrm{~cm}^{-1}$ resulted from the vibration of the tertiary nitrogen of PVP. Other observed band at $2948 \mathrm{~cm}^{-1}$ is attributed to the asymmetric stretching vibrations of $\mathrm{C}-\mathrm{H}$ bond and band at $3447 \mathrm{~cm}^{-1}$ assigned to $-\mathrm{OH}$ group due to ethylene glycol.

\section{$3.4 T E M$}

Figure 5 is a micrograph and histogram of Ir:Ni (2:1) and Ir:Ni (1:2) bimetallic nanoparticles. TEM analysis revealed that size of synthesized Ir:Ni (2:1) bimetallic nanoparticles ranges between 0.92 and $3.70 \mathrm{~nm}$, and the size of Ir:Ni (1:2) bimetallic nanoparticles ranges between 1.85 and $5.55 \mathrm{~nm}$. The spectra shows that the synthesized particles are pseudospherical with small size distribution.

\section{$3.5 \quad F E-S E M$}

The powered samples were scanned through FE-SEM, which gives information about the surface morphology of synthesized nanoparticles. FE-SEM images of synthesized bimetals at a temperature of $140-180^{\circ} \mathrm{C}$ are shown in figure 6 . From the images, it is clear that the bimetallic nanoparticles Ir:Ni (2:1) (figure $6 \mathrm{~b}$ ) have pseudo-spherical nanoparticles with nearly 

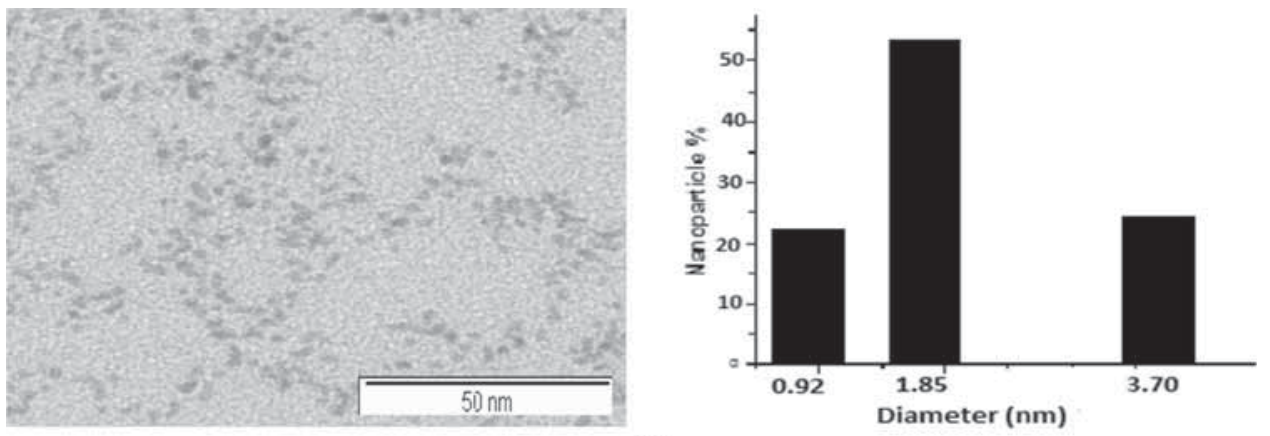

(A)
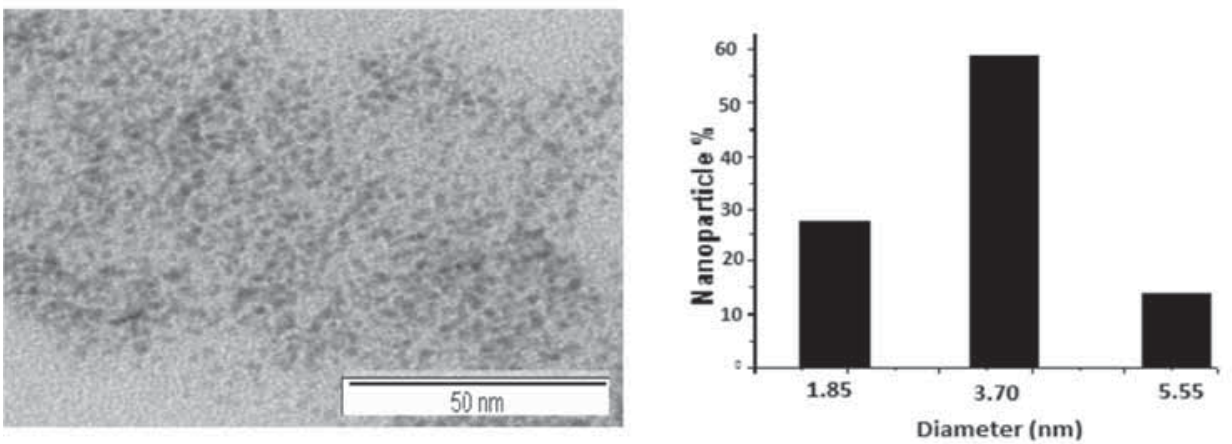

(B)

Figure 5. TEM images of (A) Ir:Ni-2:1 bimetals and (B) Ir:Ni-1:2 bimetals.
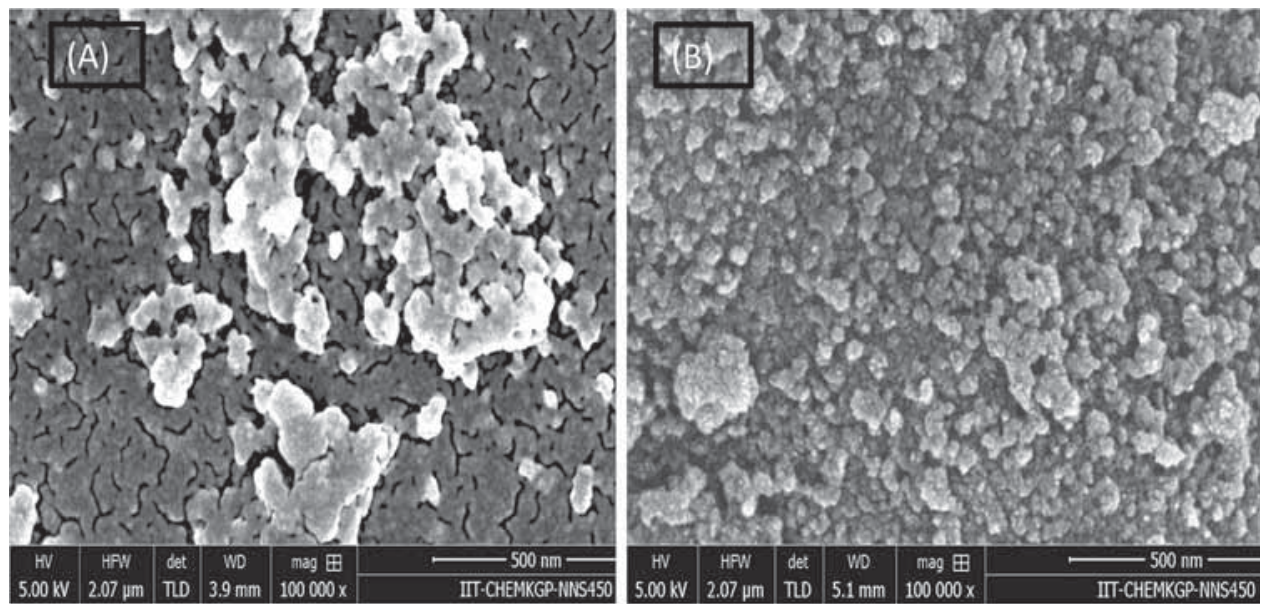

Figure 6. FE-SEM images of (A) Ir:Ni-1:2 bimetallic nanoparticless and (B) Ir:Ni-2:1 bimetallic nanoparticles.

uniform size and less dispersed in bulk state by avoiding their aggregation due to the coordination with $\mathrm{Ir}, \mathrm{Ni}$ ions in the composite mixture. Figure 6 a shows that the bimetallic nanoparticles Ir:Ni (1:2) are irregular in shape and similarly, their distribution is not uniform with some agglomeration.

\section{$3.6 X P S$}

The XPS graphs of Ir-Ni bimetallic nanoparticles for carbon, iridium and nickel are shown in figure $7 \mathrm{a}$ and $\mathrm{b}$. The oxidation state of iridium and nickel in the resulting bimetallic nanoparticles was analysed by XPS. The deconvolution of the high resolution Ir $4 \mathrm{f}$ spectrum of iridium nanoparticles gives two predominant peaks centered at 58.75 and $61.82 \mathrm{eV}$ readily assigned to $\operatorname{Ir}(0) 4 f_{7 / 2}$ and $\operatorname{Ir}(0) 4 f_{5 / 2}$, respectively, which may originated from the surface oxidation of iridium nanoparticles. The peaks at 852.2 and $851.4 \mathrm{eV}$ are the characteristic feautures of $\mathrm{Ni}^{0}\left(2 \mathrm{p}_{3 / 2}\right)$. Carbon was also detected with a nearly constant ratio from the surface to the core, and with a C1s binding energy of $282.2 \mathrm{eV}$. This peak is assigned to 

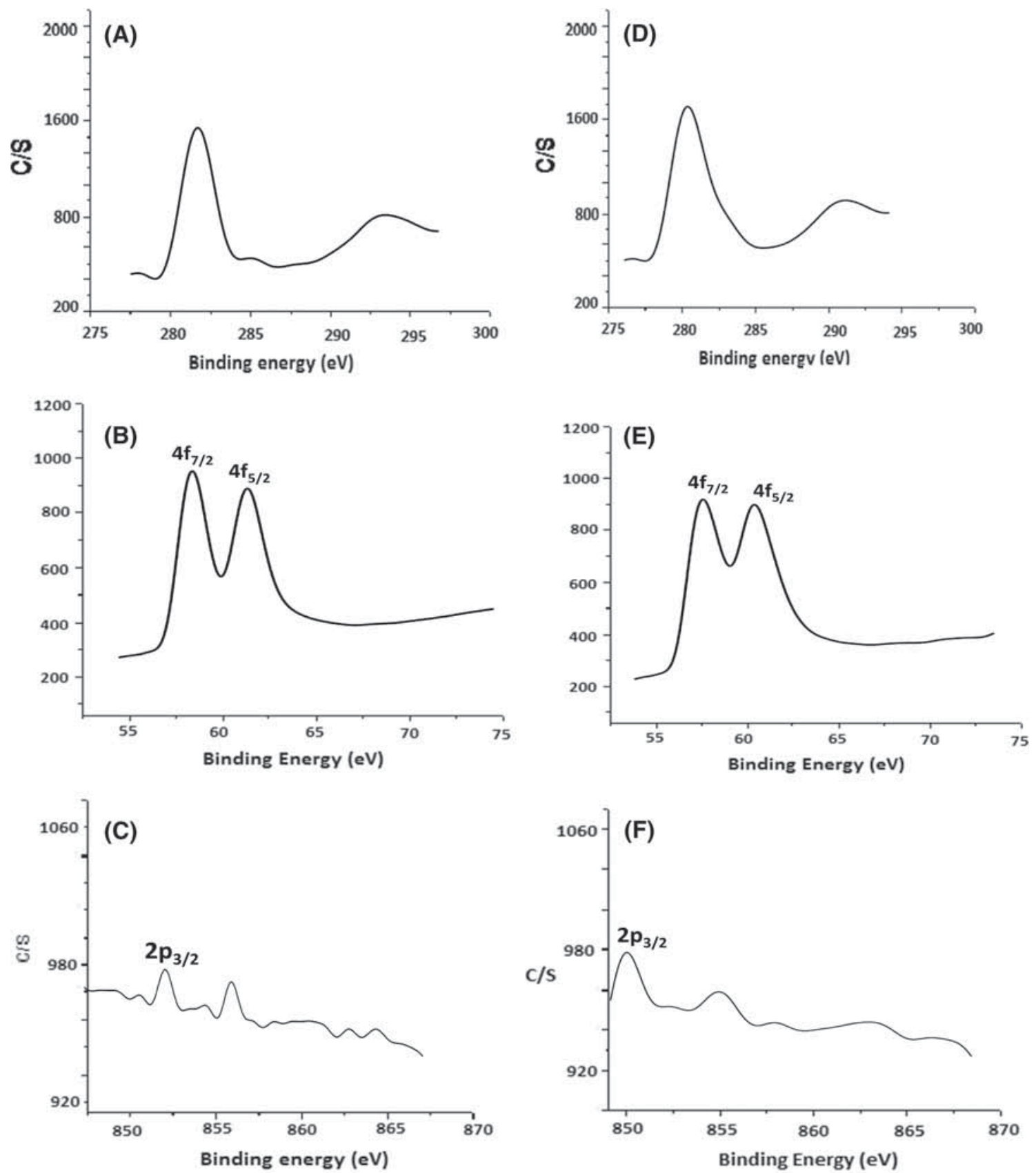

(1)

(2)

Figure 7. (a) XPS of Ir-Ni (1:2) nanoparticles for C1s, (b) XPS of Ir-Ni (1:2) nanoparticles for Ir4f, (c) XPS of Ir-Ni (1:2) nanoparticles for Ni2p, (d) XPS of Ir-Ni (2:1) nanoparticles for C1s, (e) XPS of Ir-Ni (2:1) nanoparticles for Ir4f and (f) XPS of Ir-Ni (2:1) nanoparticles for Ni2p.

carbon atoms in different environments, which may be due to PVP chain and ring [27]. The surface composition of Ir and Ni was also analysed by XPS analysis. By XPS, no change in the oxidation states of Ir, Ni and the carbon content was observed in both types of synthesized bimetallic nanoparticles. There was a single significant difference in surface atomic percent of iridium and nickle. The surface composition of Ir is high in Ir:Ni (2:1) and low in Ir: Ni (1:2).

\section{Catalytic activity of Ir-Ni bimetals for MY degradation}

The prepared Ir:Ni (2:1) and Ir:Ni (2:1) bimetallic nanoparticles were utilized as catalysts for the degradation of MY by hexacynoferrate (III) ions in aqueous alkaline medium at $\lambda_{\max }$ of reaction mixture $435 \mathrm{~nm}$. The reaction was studied at constant temprature $(40 \pm 0.1)^{\circ} \mathrm{C}$ by kinetic 


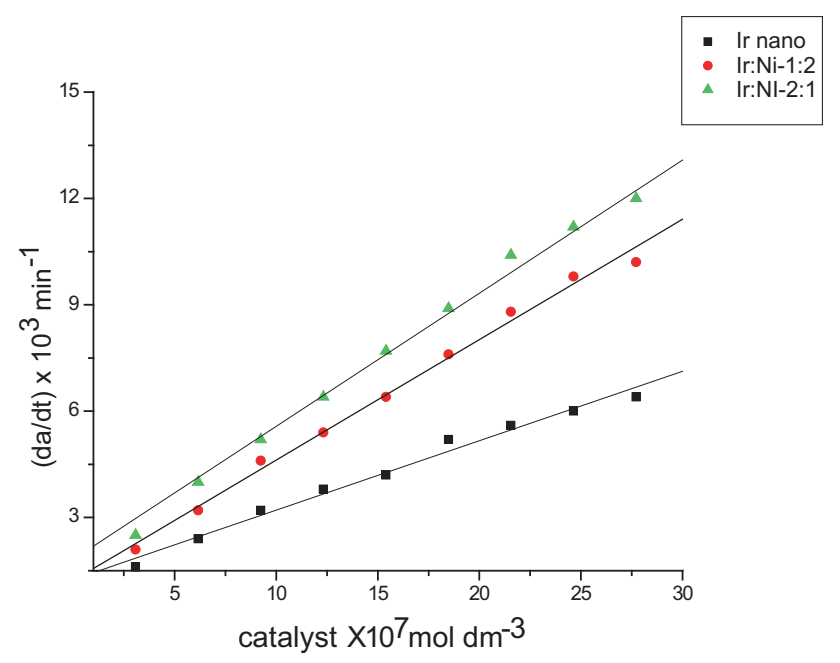

Figure 8. Rate vs. concentration of catalyst for the oxidation of metanil yellow by hexacynoferrate (III) ions.

spectrophotometric method. The comparison of data is presented in figure 8 and it shows that the addition of another metal i.e., Ni increases the catalytic activity of Ir nanoparticles. The rate of degradation of MY is highest in the presence of Ir:Ni (2:1) bimetallic nanoparticles as compared to Ir nanoparticles. The catalytic activity of Ir:Ni (1:2) bimetals is less than the catalytic activity of Ir:Ni (2:1) bimetals, which may be due to the smaller particle size of $\operatorname{Ir}-\mathrm{Ni}$ (2:1) (figure 5) providing large surface area. Furthermore, when iridium content was small, a thin primary iridium shell was found on the nickel core and as the iridium content increases, the iridium will deposit more on the vertices. So, Ir:Ni $(2: 1)$ exhibited higher catalytic activity and durability for the degradation of MY. Xu et al [28] also reported that noble metals (Rh, Pt, Ir) act as good bimetallic catalysts with $\mathrm{Ni}$ for complete decomposition of hydrous hydrazine. To study the efficiency of the catalyst i.e., Ir:Ni bimetallic nanopaticles, its catalytic activity was compared with the reported methods as presented in table 2. The data presented in the table show that though the rate of degradation of azo dyes by methods 1 and $2[11,28]$ is more than our present method, but the requirement of catalyst $\mathrm{Fe}-\mathrm{Ni}$ is about hundred times more than the $\mathrm{Ir}-\mathrm{Ni}$ bimetallic catalyst.

\section{Mechanism of dye degradation}

Based on the above experimental results and previously reported work [9], the following mechanism for oxidation of MY was proposed:

$$
\begin{aligned}
& \mathrm{MY}^{-}+(\mathrm{Ir}-\mathrm{Ni})_{n} \underset{k_{-1}}{\stackrel{k_{1}}{\rightleftharpoons}} \text { Complex }(\mathrm{C}) \\
& \mathrm{C}+\mathrm{Fe}(\mathrm{CN})_{6}^{-3} \underset{\text { Slow }}{\stackrel{k_{2}}{\longrightarrow}}(\mathrm{Ir}-\mathrm{Ni})_{n}+\mathrm{Fe}(\mathrm{CN})_{6}^{-4}+\text { Product }
\end{aligned}
$$

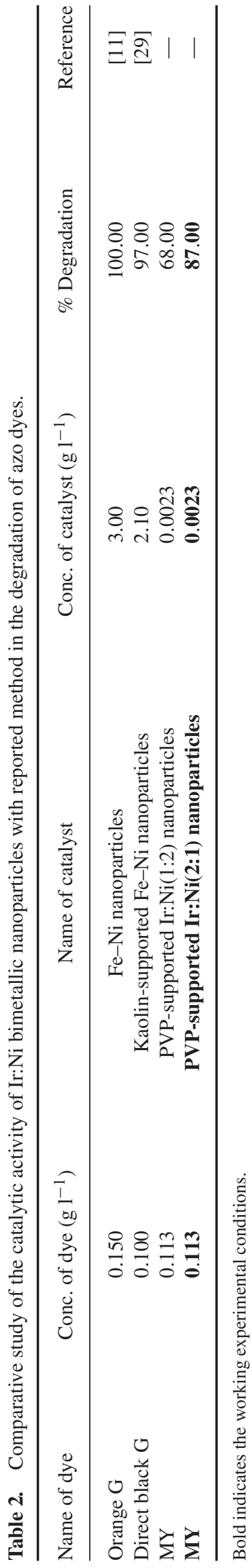




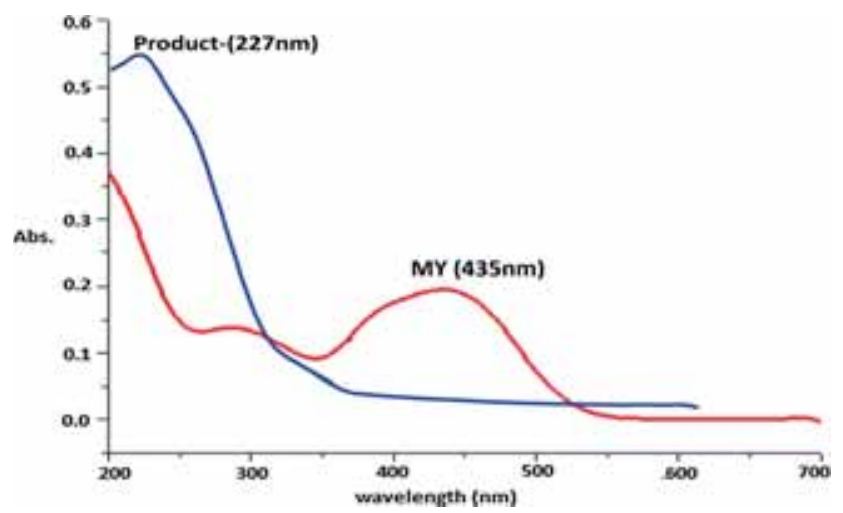

Figure 9. UV-Vis spectra of metanil yellow dye and product after degradation.

In the above mechanism, it is assumed that in alkaline medium mono azo dye, MY exists as anion $\left(\mathrm{MY}^{-}\right)$, which slowly forms a loosely bonded complex with Ir-Ni bimetallic nanoparticles $(\mathrm{Ir}-\mathrm{Ni})_{n}$. This complex $\mathrm{C}$ slowly reacts with $\mathrm{HCF}(\mathrm{III})$ ion resulting into a product along with $(\mathrm{Ir}-\mathrm{Ni})_{n}$ and $\mathrm{Fe}(\mathrm{CN})_{6}^{-4}$.

Degradation of MY was monitored by UV-Vis spectroscopy (figure 9) in which MY is characterized by maximum adsorption at $435 \mathrm{~nm}$ attributed to the chromophore containing azo linkage of the dye molecule in the solution. The disappearance of the band and formation of new band at $227 \mathrm{~nm}$ supports the degradation of MY due to the breakdown of azo linkage.

\section{Conclusion}

PVP-supported Ir:Ni (2:1) and Ir:Ni (1:2) bimetallic nanoparticles with the average particle size of 1.85 and $3.70 \mathrm{~nm}$, respectively, and can be synthesized easily by the polyol method, which remains stable even after several months. TEM analysis confirms that the as-prepared nanoparticles are small in size with less-dispersed size distribution. The XRD studies also reveal that the bimetallic nanoparticles predominantly assemble in FCC structure. The attachment of PVP on the surface of bimetals $(0)$ nanoparticles through the $\mathrm{C}=\mathrm{O}$ groups was concluded from the observation of change in $\mathrm{C}=\mathrm{O}$ stretching frequency of PVP in the FT-IR spectra. The oxidation state of the resulting nanoparticles was determined by XPS measurements. The synthesized bimetallic nanoparticles were utilized as catalyst for the reduction of MY by hexacynoferrate (III) ions and the rate constants for the degradation reaction were determined and compared. It was found that the bimetallic (Ir:Ni 2:1) nanoparticles increase the rate of degradation of MY more as compared to bimetallic ( $\mathrm{Ir}: \mathrm{Ni} 1: 2)$ nanoparticles and the iridium monometallic nanoparticles which may be attributed to the synergistic effects of the two monometallic nanoparticles i.e., bimetallization improves the properties of metal nanoparticles. These bimetallic nanoparticles can be utilized to degrade various other toxic dyes which are decanted off and are very harmful to our environment.

\section{Acknowledgements}

We would like to express our sincere gratitude to IIT, Roorkee for XRD analysis, IIT, Kanpur for XPS and TEM analyses and IIT, Kharagpur for FE-SEM analysis.

\section{References}

[1] Shanker U, Rani M and Jassal V 2017 Environ. Chem. Lett. https://doi.org/10.1007/s10311-017-0650-2

[2] Pal T, Kundu S, Panigrahi U, Pal A, Ghosh S K, Nath S et al 2006 Dyes Pigments 69177

[3] Vinodgopal K and Peller J 1998 Water Res. 321425

[4] Belachew N and Devi D R 2017 J. Exp. Nano Sci. 12114

[5] Raffi F, Hall J D and Cerniglia C E 1997 Food Chem. Toxicol. 35897

[6] Singh R L 1989 Biochem. Int. 191109

[7] Forgacs E, Cserha T and Oros G 2004 Environ. Int. 30953

[8] Furlan F R, Silva L G and Morgado A F 2010 Res. Con. Recycl. 54283

[9] Goel A and Lasyal R 2016 D. Water Treat. 5717547

[10] Thomas J M, Raja R, Johnson B F G, Hermans S, Jones M D and Khimyak T 2003 Int. Eng. Chem. Res. 421563

[11] Bokare A D, Chikate R C, Rode C V and Paknikar K M 2008 Appl. Catal. B: Environ. 79270

[12] Mulvaney P, Giersig M and Henglein A 1992 J. Phys. Chem. 964329

[13] Singh A K and Xu Q 2013 Chem. Cat. Chem. 5652

[14] Yadav M and Xu Q 2012 Energy Environ. Sci. 59698

[15] Jiang H L, Singh S K, Yan J M, Zhang X B and Xu Q 2013 Chem. Sus. Chem. 5541

[16] Kundu S, Mukadam M D, Yusuf M S and Jayachandran M 2013 Cryst. Eng. Comm. 15482

[17] Goel A and Rani N 2012 Open J. In. Chem. 267

[18] Yang H, Vogel W, Lamy C and Vante N A 2004 J. Phys. Chem. B 10811024

[19] Narayanan R and El-Sayed M A 2005 J. Phys. Chem. B 109 12663

[20] Singha H P, Gupta N, Sharmab S K and Sharma R K 2013 Physicochem. Eng. Aspects 41643

[21] Baranova E A, Amir T, Mercier P H J, Patarachao B and Wang D 2010 J. Appl. Electrochem. 401767

[22] Garcia-Gutierrez D L and Gutierrez-Wing C E 2005 J. Phys. Chem. B 1093813

[23] Su H, Liu D D, Zhao M et al 2015 ACS Appl. Mater. Interf. 7 8233

[24] Chandra S, Kumar A and Tomar P A 2014 J. Saudi Chem. Soc. 18437

[25] Sasaki K, Kuttiyiel K A and Barrio D 2011 J. Phys. Chem. C 1159894

[26] Goel A and Bhatt R 2012 Int. J. Chem. App. 2111

[27] Couto G G, Klein J J, Schreiner W H, Mosca D H, Oliveira A J A and Zarbin A J G 2007 J. Colloid Int. Sci. 311461

[28] Singh A K and Xu Q 2014 Int. J. Hydrogen Energy 399128

[29] Liu X, Chen Z, Megharaj M and Naidu R 2013 Chem. Eng. J. 223764 